\title{
Single-particle absorption spectroscopy by photothermal contrast
}

\author{
Mustafa Yorulmaz ${ }^{1,+}$, Sara Nizzero ${ }^{2,3,+}$, Anneli Hoggard ${ }^{1}$, Lin-Yung Wang $^{1}$, \\ Yiyu Cai ${ }^{1}$, Man-Nung Su ${ }^{1}$, Wei-Shun Chang ${ }^{1}$, Stephan Link ${ }^{1,3, *}$ \\ ${ }^{1}$ Department of Chemistry, ${ }^{2}$ Applied Physics Graduate Program, ${ }^{3}$ Department of Electrical and \\ Computer Engineering, Laboratory for Nanophotonics, Rice University, Houston, Texas 77005, \\ United States \\ $\$$ These authors contributed equally to this work \\ * Corresponding author’s e-mail: slink@rice.edu
}




\begin{abstract}
Removing effects of sample heterogeneity through single-molecule and single-particle techniques has advanced many fields. While background free luminescence and scattering spectroscopy is widely used, recording the absorption spectrum only is rather difficult. Here we present an approach capable of recording pure absorption spectra of individual nanostructures. We demonstrate the implementation of single-particle absorption spectroscopy on strongly scattering plasmonic nanoparticles by combining photothermal microscopy with a supercontinuum laser and an innovative calibration procedure that accounts for chromatic aberrations and wavelength-dependent excitation powers. Comparison of the absorption spectra to the scattering spectra of the same individual gold nanoparticles reveals the blueshift of the absorption spectra, as predicted by Mie theory but previously not detectable in extinction measurements that measure the sum of absorption and scattering. By covering a wavelength range of $300 \mathrm{~nm}$, we are furthermore able to record absorption spectra of single gold nanorods with different aspect ratios. We find that the spectral shift between absorption and scattering for the longitudinal plasmon resonance decreases as a function of nanorod aspect ratio, in agreement with simulations.
\end{abstract}

Keywords: Single-particle spectroscopy, absorption spectroscopy, photothermal imaging, darkfield scattering spectroscopy, gold nanoparticle, gold nanorod, surface plasmon 
Single-molecule fluorescence spectroscopy ${ }^{1}$ had a tremendous impact in materials science, ${ }^{2}$ biophysics, ${ }^{3}$ and quantum optics ${ }^{4}$ as it removes sample heterogeneity and resolves competing kinetic pathways. ${ }^{5}$ Many molecules and nanoparticles are, however, not luminescent. ${ }^{6-8}$ Moreover, the ability to correlate absorption and fluorescence spectra of the same sample offers the fascinating possibility to unravel the mechanisms underlying photoblinking and photobleaching of single-molecules, ${ }^{5}$ or the nature of absorptive vs. emissive centers in conjugated polymers. ${ }^{9,}$ Although absorption-based imaging has recently been demonstrated. ${ }^{11-}$ ${ }^{18}$ no absorption spectroscopy is available for nanostructures of arbitrary size, geometry, and material. Absorption spectroscopy of single molecules, complex nanostructures, and macromolecular systems would be a significant advancement with great impact as, for example, the identification of degenerate intermediates in enzymatic and catalytic processes have so far only been examined on the ensemble level. ${ }^{19}$

Recording the pure absorption spectrum of single molecules and nanoparticles at room temperature is difficult by standard transmission-type extinction measurements because: 1) the reduction in concentration to a single object yields a signal that is typically too small to measure on the high background signal of the incident light unless ultrasmooth surfaces and ultrasensitive photodetectors $^{12,20}$ are used; 2) even if the size of the object is increased to enhance the absorption, typically scattering is then also present and contributes to the extinction signal. The first single-molecule optical detection experiment was achieved by absorption using a doublemodulation technique, but had to be carried out at cryogenic conditions, under which the absorption cross-section of the zero-phonon line is $10^{6}$ times larger. ${ }^{21}$ Indirect detection schemes combined with modulation techniques have been developed for absorption-based detection, ${ }^{22-25}$ 
including ground-state depletion microscopy ${ }^{15,26}$ and photothermal microscopy. ${ }^{11,13,14,16,27-30}$ However, most studies using these methods have reported single-wavelength imaging without yielding spectroscopic information. A tunable dye laser, incorporated as the excitation source into a photothermal microscope, has allowed the acquisition of absorption, albeit in a limited spectral range of $515 \mathrm{~nm}$ to $585 \mathrm{~nm}$, for which chromatic aberrations were mostly negligible. ${ }^{14}$, 31, 32 Single-particle extinction techniques, such as spatial modulation spectroscopy ${ }^{24,33,34}$ and white-light scattering interference spectroscopy, ${ }^{35}$ have been applied as an alternative route to record the spectrum of nanoparticles. ${ }^{35,36}$ Although for the smallest nanoparticle sizes the extinction spectrum is dominated by absorption, it is often important to obtain the pure absorption spectrum, ideally in comparison to the scattering of the same nanoparticle. ${ }^{37}$ To date this knowledge is often only accessible through simulations, even though it has important implications for many applications ranging from cancer therapy ${ }^{38}$ to hot electron generation ${ }^{39}$ and light harvesting. ${ }^{40}$ In particular, the absorption and scattering spectra of coupled nanostructures that support more complex plasmon modes can differ significantly from each other. For example, the scattering spectrum of a cluster of nanoparticles supporting a Fano resonance shows a pronounced dip indicative of interference of bright and dark modes, while the corresponding absorption spectrum reaches a maximum value close to the same wavelength. ${ }^{41}$ 

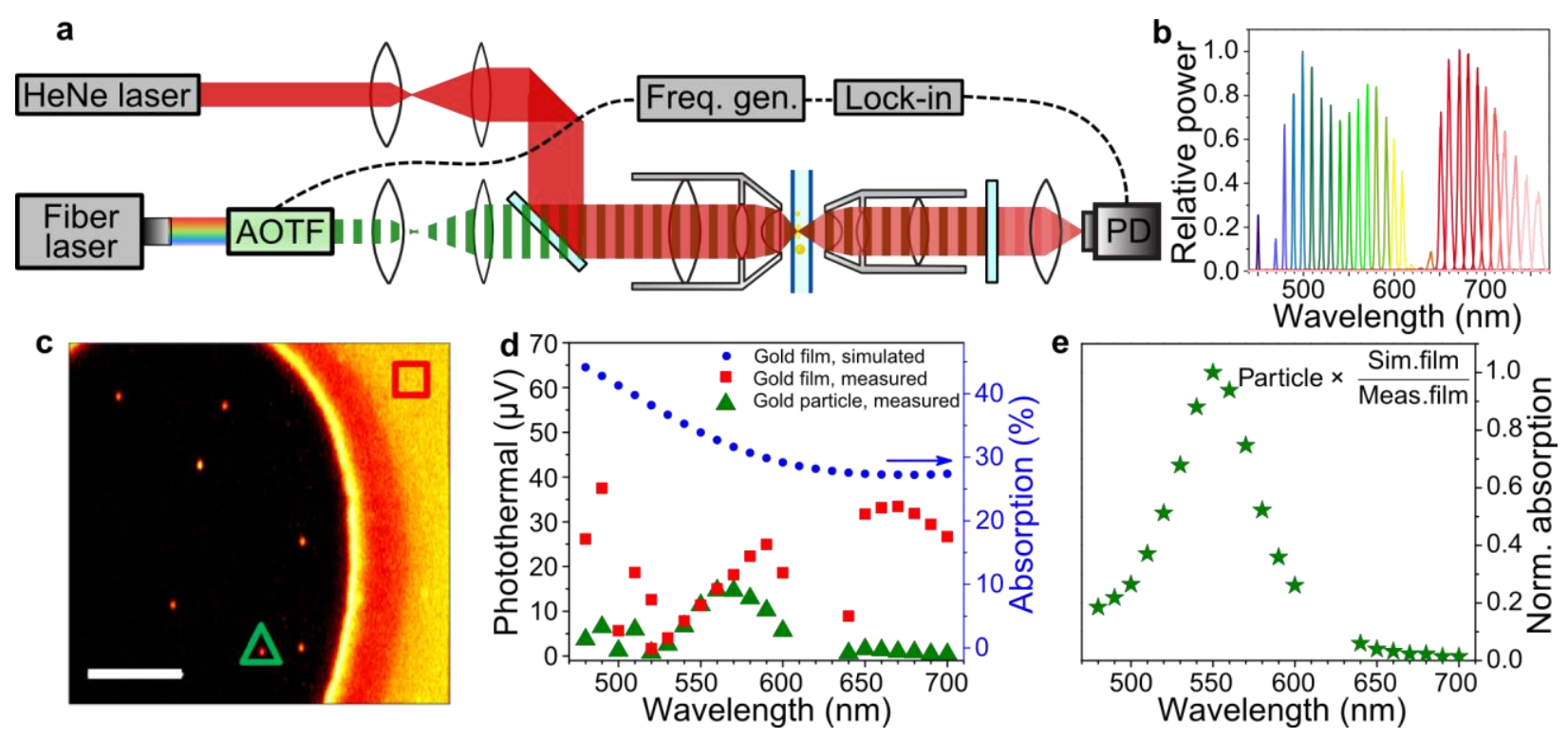

Figure 1. Principle of single-particle absorption spectroscopy. a, Illustration of photothermal microscopy in transmission geometry employing a broadband laser (Fianium Whitelase SC450) equipped with an AOTF. b, Relative laser powers of different wavelengths selected using the AOTF and measured before entering the microscope with a fiber-coupled spectrometer (Ocean Optics USB4000). The spectral sensitivity of the spectrometer is accounted for by recording the power for each laser line with a calibrated optical energy meter (Thorlabs PM100D). The gap in the spectral range from $\sim 610 \mathrm{~nm}$ to $\sim 630 \mathrm{~nm}$ is due the limited transmission of the dichroic (Chroma Technology Corp., ZQ633RDC) used to combine heating and probe beams. c, Typical photothermal image of $50 \mathrm{~nm}$ gold nanoparticles (round spots) and the $15 \mathrm{~nm}$ thin gold film (large uniform-signal area). Experimental parameters are: heating beam intensity at $550 \mathrm{~nm}=$ $36 \mathrm{~kW} / \mathrm{cm}^{2}$; probe beam intensity at $633 \mathrm{~nm}=0.27 \mathrm{MW} / \mathrm{cm}^{2}$; integration time of lock-in amplifier $=10 \mathrm{~ms}$; modulation frequency $=230 \mathrm{kHz}$. Except for the heating and probe beam intensity these parameters are the same for all experiments. The scale bar is $5 \mu \mathrm{m}$. d, Measured photothermal signal as a function of wavelength for the gold film (•) and the nanoparticle ( $\Delta$ ) highlighted in $\mathbf{c}$. The calculated absorption spectrum of the gold film using FDTD simulations (•) 
is also shown. e, The corrected spectrum $(\star)$ of the same gold nanoparticle as in $\mathbf{c}$ and $\mathbf{d}$ obtained via a correction factor based on the ratio of simulated and measured film absorption.

Here we demonstrate the implementation of single-particle absorption spectroscopy on strongly scattering plasmonic nanoparticles. Pure absorption spectra are obtained through the incorporation of a supercontinuum laser as the heating beam into a standard photothermal imaging setup employing a transmission geometry. A coherent supercontinuum light source has already been successfully combined with spatial modulation spectroscopy. ${ }^{25}$ In addition, we describe a simple correction method that accounts for the unavoidable effects of chromatic aberrations, necessary in our case because of the much larger wavelength range $(300 \mathrm{~nm})$ than explored before. We are therefore able to acquire absorption spectra of anisotropic nanostructures with multiple plasmon modes. The advantage of our approach is that we are able to measure absorption only free from scattering.

Photothermal spectroscopy is carried out in transmission geometry (Fig. 1a, see Supporting Information for details). Excitation wavelengths with $3-6 \mathrm{~nm}$ bandwidth (Fig. 1b) are selected from a supercontinuum laser $(450-2000 \mathrm{~nm})$ with an acousto-optical-tunable filter (AOTF), which also provides the time-dependent modulation necessary for sensitive lock-in detection of the photothermal signal by a $633 \mathrm{~nm}$ probe beam. The photothermal signal at each excitation wavelength is proportional to its absorption cross-section, but also depends on the heating beam power and size as well as the 3D overlap of the heating and probe beams at the focus. All of these parameters change with the excitation wavelength because of the wavelength-dependent power output of the laser (Fig. 1b) and the use of diffractive optics that cause inevitable 
chromatic aberrations. While adjustments for intensity variations and aberrations could in principle be made for each wavelength, and potentially even be automated, these issues severely hinder the straightforward implementation of photothermal spectroscopy, especially for high throughput measurements.

We can solve this problem though, and successfully record the absorption spectrum of plasmonic nanoparticles using a calibration approach akin to dark-field spectroscopy where the measured nanoparticle spectrum is divided by the scattering from the nearby substrate. ${ }^{42}$ Our simple calibration procedure involves the measurement of a single nanoparticle followed by an adjacent gold film (Fig. S1), as demonstrated in Figs 1c - 1e for nominal $50 \mathrm{~nm}$ diameter gold nanoparticles (independent characterization: $49 \pm 4 \mathrm{~nm}$, Fig. S2). We first image the sample at a fixed wavelength of $550 \mathrm{~nm}$ to identify the positions of individual gold nanoparticles (bright spots on a dark background in Fig. 1c) and the $15 \mathrm{~nm}$ thin gold film (extended bright area). After positioning the scanning stage to a nanoparticle, we continuously record the photothermal signal as a function of time while automatically changing the heating beam wavelength in $10 \mathrm{~nm}$ increments each $10 \mathrm{~s}$ without any other adjustments (Fig. S3). Conversion of this transient into a spectrum results in the distorted lineshape shown in Fig. 1d. This procedure is then repeated for the film under the same experimental conditions. Comparison of the measured film spectrum with the calculated absorption using finite difference time domain (FDTD) simulations (Fig. 1d and Fig. S4) provides a simple, reproducible, and precise correction factor, which, when applied to the measured nanoparticle spectrum, gives the corrected absorption spectrum (Fig. 1e). 
A variety of control experiments demonstrate the validity of our correction procedure. Experiments using an aluminum film instead works equally well (Fig. S7). The signal intensity and line shape of the film spectrum are furthermore highly reproducible when measured at different positions (Fig. S8a). The corresponding corrected nanoparticle absorption spectra using either of the measured film spectra are hence in excellent agreement (Fig. S8b), confirming that possible small variations in the thickness and local imperfections of the gold film do not affect the correction. However, the thickness of the gold film for different samples can vary slightly. Therefore, the film thickness of each sample is independently characterized with atomic force microscopy (AFM, Fig. S5) and the corresponding film absorption spectrum is simulated and used in the correction procedure. In addition, the geometry of the thermal lens created by heating of the continuous film in comparison to the nanoparticle could be different within the area of the probe laser $^{43,44}$ and change the relative magnitudes of the measured signals. However, the absorption spectra of the same nanoparticle measured using excitation at different angles (Fig. S9) show little dependence on the thermal lens geometry. Finally, absorption spectra recorded after optimizing the signal in both radial and axial directions at different wavelengths (Fig. S6) are the same within the experimental error, indicating that small wavelength-dependent migrations of the focal spot do not play a role.

Although the use of high repetition rate picosecond pulses as the heating beam causes a slight decrease in photothermal sensitivity compared to continuous wave (cw) excitation, we are able to record absorption spectra of nominal $20 \mathrm{~nm}$ gold nanoparticles (independent characterization:

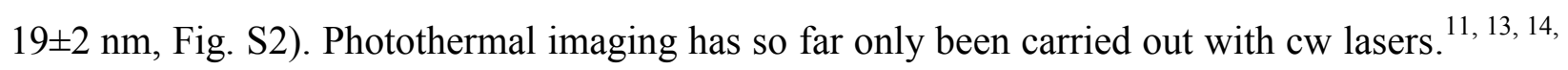
${ }^{16}$ However, a supercontinuum laser requires high peak power pulses to generate white light in a photonic fiber. For pulsed excitation of plasmonic nanoparticles with the same average laser 
power, heat diffusion and initial transient temperature increase at the nanoparticle surface are different. ${ }^{45}$ We therefore characterized the spatial and axial photothermal profiles (Figs. 2a and 2b) and signal-to-noise ratios (SNR, Figs. $2 \mathrm{c}$ and $2 \mathrm{~d}$ ) of $20 \mathrm{~nm}$ nanoparticles using $532 \mathrm{~nm} \mathrm{cw}$ and pulsed excitation. The spatial and axial resolutions for pulsed illumination are larger than the ones measured with cw excitation (Figs. $2 \mathrm{a}$ and $2 \mathrm{~b}$ ). While we can rule out that this difference is due to variations in the axial overlap of heating and probe beams or caused by underfilling the objective with the pulsed heating beam (see Supporting Information for details), it is possible that the laser light emerging from the fiber and AOTF is not of a pure $\mathrm{TEM}_{00}$ mode at the sample. Indeed, the resolution of confocal sample scanned luminescence images is similarly decreased when using the supercontinuum laser for excitation (not shown). Also, the SNR is smaller by a factor of about 2.5 for pulsed excitation for the same average power (Figs. $2 \mathrm{c}$ and 2d), likely because of an overall reduced heating given the $16.67 \mathrm{~ns}$ dark periods between pulses. Nevertheless, our sensitivity with tunable pulsed excitation is still comparable to the limits set in the literature, ${ }^{29}$ and we are able to not only image an individual $20 \mathrm{~nm}$ gold nanoparticle, but also record its absorption spectrum, in very good agreement with Mie theory calculations that include 10 multipolar modes (Fig. 2e). ${ }^{46}$ We only observe a slight mismatch between the experimental absorption spectra and Mie theory calculations at wavelengths shorter than $\sim 500 \mathrm{~nm}$ where interband transitions contribute to the spectrum. The photothermal signal is consistently lower than the calculated absorption for all gold nanoparticles investigated. The origin of this small deviation is not clear at the moment and will be investigated further in the future. 

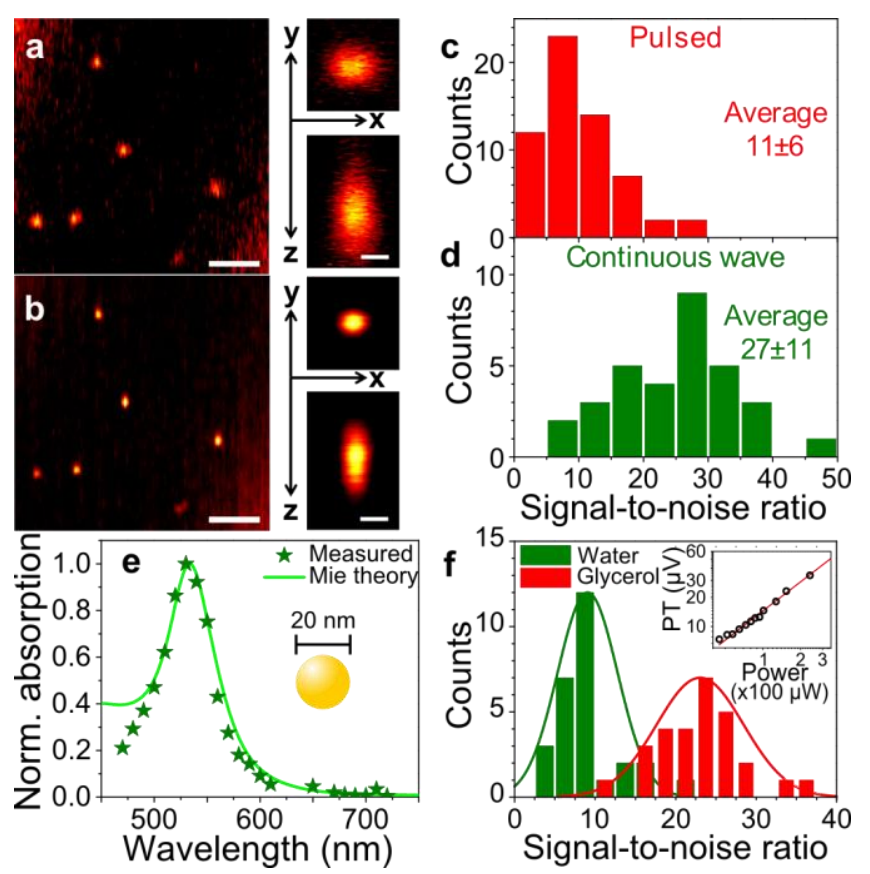

Figure 2. Photothermal microscopy using pulsed excitation. a, Photothermal images of $20 \mathrm{~nm}$ gold nanoparticles with spatial and axial profiles recorded using supercontinuum pulsed laser excitation at $532 \mathrm{~nm}$ with a heating intensity of $25 \mathrm{~kW} / \mathrm{cm}^{2}$. The spatial and axial resolutions are $\sigma_{\mathrm{x}}=340 \mathrm{~nm}, \sigma_{\mathrm{y}}=470 \mathrm{~nm}$, and $\sigma_{\mathrm{z}}=720 \mathrm{~nm}$. b, Same sample area as in a but measured with cw laser excitation at $532 \mathrm{~nm}$ with a heating intensity of $38 \mathrm{~kW} / \mathrm{cm}^{2}$ (scale bar is $2 \mu \mathrm{m}$ ); $\sigma_{\mathrm{x}}=220 \mathrm{~nm}$, $\sigma_{\mathrm{y}}=250 \mathrm{~nm}$, and $\sigma_{\mathrm{z}}=560 \mathrm{~nm}$ (scale bars are $300 \mathrm{~nm}$ ). c, SNR of the photothermal signal for 55 nanoparticles measured with pulsed excitation. d, SNR of the photothermal signal for 32 nanoparticles measured with cw excitation. The SNRs shown in $\mathbf{c}$ and $\mathbf{d}$ are normalized to the same heating power and obtained on $20 \mathrm{~nm}$ gold nanoparticles. e, Absorption spectrum of a single $20 \mathrm{~nm}$ diameter gold nanoparticle embedded in glycerol measured using a heating laser intensity of $11 \mathrm{~kW} / \mathrm{cm}^{2}$ at $550 \mathrm{~nm}$ in comparison to Mie theory calculations. A weighted average of the refractive indices of glycerol and glass (1.495) and a nanoparticle size within the experimental size dispersion (Fig. S2) are assumed. Excellent agreement is found for a size of $20 \mathrm{~nm}$. f, Effect of the embedding medium on the photothermal signal of $50 \mathrm{~nm}$ diameter 
nanoparticles. The SNR ratio increases from $9 \pm 4$ to $23 \pm 5$ when changing the medium from water to glycerol. Inset shows that the power dependence of the photothermal signal (PT) at $550 \mathrm{~nm}$ under pulsed laser excitation is linear.

We furthermore confirm the photothermal nature of the signal to rule out other contributions, such as electronic transitions. ${ }^{47}$ Because the photothermal signal is proportional to the change in the medium's refractive index per temperature increase and to the heating power, ${ }^{13,}{ }^{29}$ we compare the SNR of the same nanoparticles in different media and perform a power dependence. The results for different media are consistent with previous cw photothermal imaging (Fig 2f). ${ }^{29}$ Changing the excitation power by a factor of 3 furthermore does not result in significant broadening of the plasmon resonance (Fig. S10), as would be expected for an electronic signal. ${ }^{48}$ The laser power dependence of the photothermal signal (see inset of Fig. $2 \mathrm{f}$ and Fig. S11 for additional wavelengths including data for $<500 \mathrm{~nm}$ ) at selected wavelengths shows the expected linear relationship for all wavelengths within the power regime tested and furthermore confirms the absence of significant spectral broadening in our absorption spectroscopy experiments. Greater care must, however, be taken though with pulsed excitation to not increase the excitation power beyond a size specific threshold as we then observe the disappearance of the signal likely due to desorption of the nanoparticle from the surface caused possibly by increased radiation pressure. 


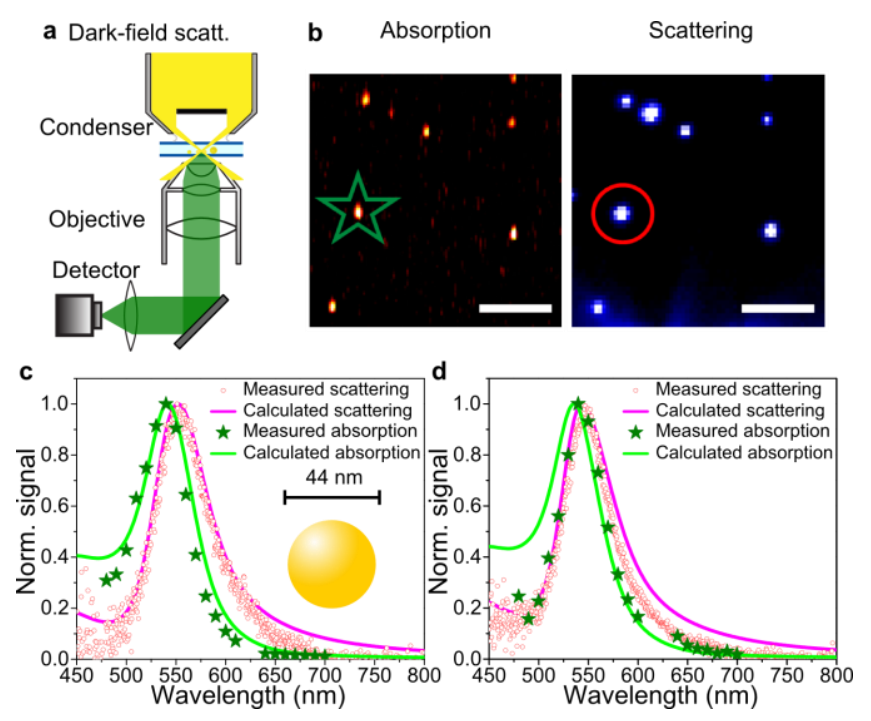

Figure 3. Absorption and scattering spectroscopy of $50 \mathrm{~nm}$ gold nanoparticles. a, Schematic drawing of conventional dark-field spectroscopy used to record the scattering spectrum of single gold nanoparticles for direct comparison with absorption measurements of the same individual nanoparticles. b, Correlation of absorption and scattering images of $50 \mathrm{~nm}$ gold nanoparticles. The scale bar is $5 \mu \mathrm{m}$. c, Correlated absorption and scattering spectra of the nanoparticle highlighted by $i$ and $\circ$ in the images. The experimental absorption and scattering spectra are

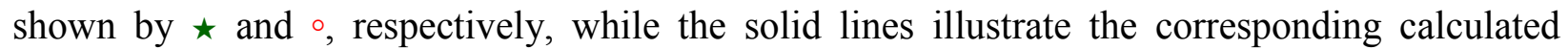
spectra obtained using Mie theory for a nanoparticle diameter within the experimental size distribution (Fig. S2) and an effective refractive index of 1.495 for nanoparticles on glass and embedded in glycerol. Excellent agreement is found between experimental and theoretical spectra for a nanoparticle size of $44 \mathrm{~nm}$. $\mathbf{d}$, The same nanoparticle as in c but embedded in water. The measured absorption and scattering spectra cannot be reproduced by Mie theory, probably due to local inhomogeneities of the medium around the nanoparticle. The average heating laser intensity used to record the absorption images and the spectra is $20 \mathrm{~kW} / \mathrm{cm}^{2}$ at $550 \mathrm{~nm}$. 
As photothermal imaging measures pure absorption only, a major benefit of our spectroscopy method is that it can distinguish the absorption and scattering spectra of larger nanoparticles, information that otherwise is hidden in extinction. We demonstrate this advantage here by comparing the absorption and scattering spectra of the same $50 \mathrm{~nm}$ gold nanoparticles using dark-field spectroscopy (Fig. 3a, see Supporting Information for details ) and correlated imaging with indexed substrates (Figs. $3 \mathrm{~b}$ and S1). ${ }^{49}$ Fig. $3 \mathrm{c}$ shows the measured absorption and scattering spectra, which are in excellent agreement with Mie theory calculations. ${ }^{46}$ Importantly, we observe, for the first time experimentally, the expected blueshift of the absorption spectrum for a plasmonic nanoparticle. ${ }^{37}$ The spectral shift is about $9 \mathrm{~nm}$. Among 15 nanoparticles measured though not all of them show the same match with theory (Fig. 3d), indicative of local heterogeneities in the nanoparticle shape and its nanoenvironment as well as substrate effects. ${ }^{49}$ The nanoparticle in Fig. 3d is an example of a nanoparticle for which Mie theory cannot match the absorption and scattering spectra. This nanoparticle is the same as in Fig. 3c, but covered with water. Microscope stability over the time course of an absorption spectrum (Fig. S13) and issues with measurement reproducibility (average error of $\sim 10 \%$, Fig. S14) can be ruled out as possible explanations. 

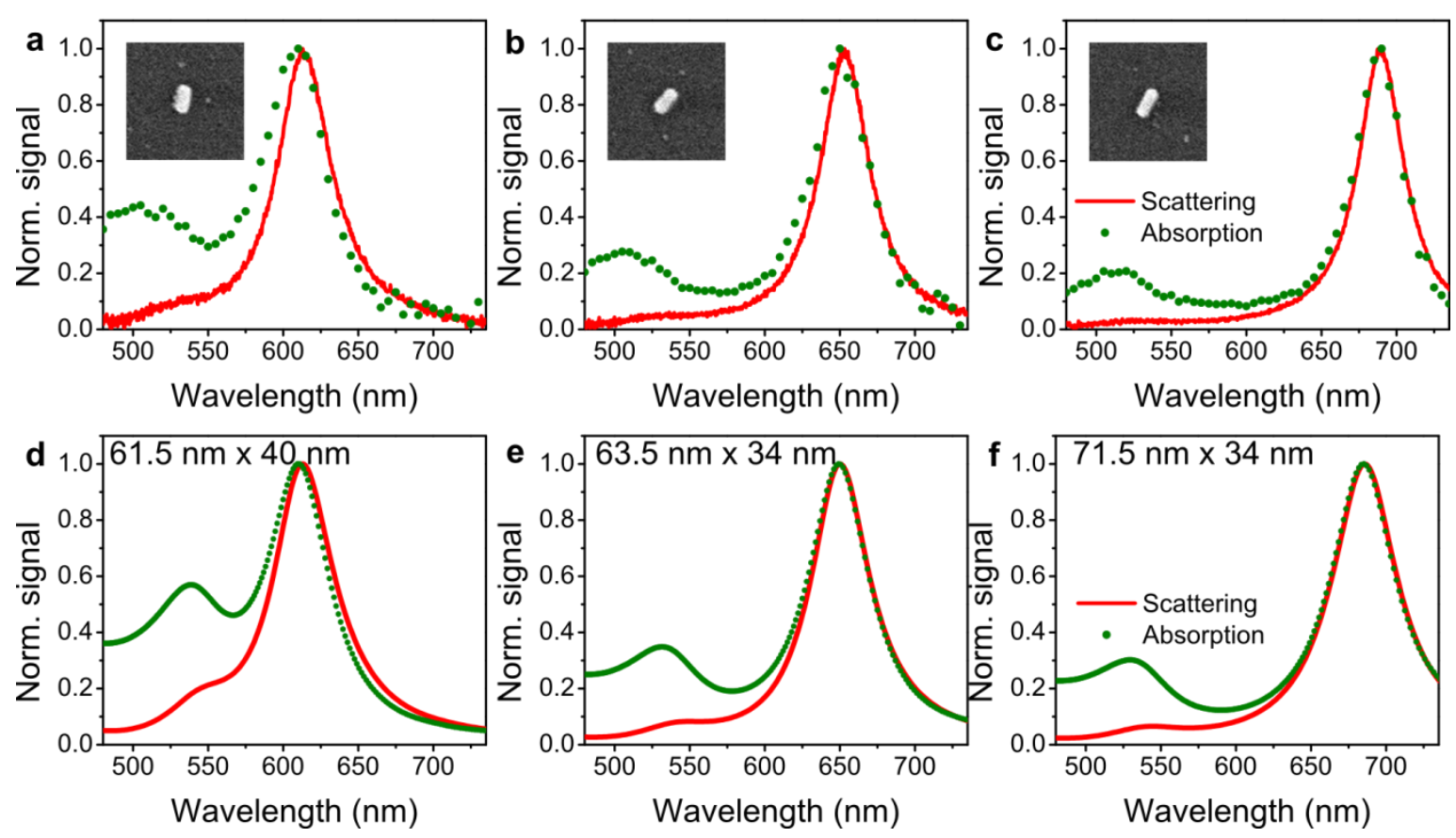

Figure 4. Absorption spectra of individual gold nanorods correlated with scattering, morphology and simulations. a, b, and $\mathbf{c}$ are measured $\left({ }^{\bullet}\right.$ )absorption spectra, correlated with measured scattering (一) spectra on the same nanorods. The experiments are repeated for nanorods with different scattering (absorption) plasmon resonances of $610.6 \mathrm{~nm}(606.8 \mathrm{~nm}), 652.0 \mathrm{~nm}$ $(649.6 \mathrm{~nm})$ and $687.8 \mathrm{~nm}(687.7 \mathrm{~nm})$ for a, b, and c, respectively. The correlated SEM images of the nanorods are shown in the insets of $a, b$, and c (the size of each image is $250 \mathrm{~nm}$ ). Using the SEM images, the lengths and widths of the nanorods are determined as $61.5 \mathrm{~nm} \times 40 \mathrm{~nm}$, $63.5 \mathrm{~nm} \times 34 \mathrm{~nm}$, and $71.5 \mathrm{~nm} \times 34 \mathrm{~nm}$ for the nanorods in a, b, and c, respectively. These sizes are used to perform FDTD simulations with the results given shown in $\mathbf{d}, \mathbf{e}$, and $\mathbf{f}$. The calculated scattering (absorption) plasmon resonances in $\mathrm{d}$, e, and $\mathrm{f}$ are $613.4 \mathrm{~nm}(608.9 \mathrm{~nm}), 650.6 \mathrm{~nm}$ $(649.0 \mathrm{~nm})$, and $685.8 \mathrm{~nm}(684.7 \mathrm{~nm})$. The measured (calculated) peak shift between absorption and scattering for the longitudinal plasmon resonance is $3.8 \mathrm{~nm}(4.5 \mathrm{~nm}), 2.4 \mathrm{~nm}(1.6 \mathrm{~nm})$, and $0.1 \mathrm{~nm}(1.1 \mathrm{~nm})$. The average laser intensity at $500 \mathrm{~nm}$ is $\sim 51 \mathrm{~kW} / \mathrm{cm}^{2}$. Individual gold nanorods 
are deposited and immobilized on a glass substrate with gold film pattern by spin-coating and then embedded in glycerol.

The broad wavelength tunability of the supercontinuum laser source as compared to previously used dye lasers ${ }^{14}$ allows us to extend our single-particle absorption spectroscopy method to other plasmonic nanoparticle shapes. Absorption spectra of single gold nanorods with different aspect ratios are compared to scattering spectra in Fig. 4a-c (see Fig. S15 and S16 for the size characterization of the nanorod samples). To record the entire absorption spectrum without a gap in the spectrum for different nanorods, we replace the $632 \mathrm{~nm}$ probe laser with a $785 \mathrm{~nm}$ laser and switch accordingly the bandpass filter in the detection path of our photothermal microscope. Scattering spectra are measured before and after photothermal spectroscopy to check whether photothermal heating has led to a change in nanorod morphology. Any nanorods showing a blueshift of the longitudinal plasmon scattering indicative of melting are discarded from the analysis. Creating circular polarized excitation over a broad wavelength is difficult, and we therefore perform the single-particle absorption spectroscopy of each nanorod with two orthogonal polarizations by simply rotating the sample by 90 degrees. The two orthogonal polarized spectra are then averaged to obtain the final 'unpolarized' absorption spectrum that shows no bias for nanorod orientation. Focusing on the longitudinal plasmon resonance, a clear blueshift of the absorption maximum is seen compared to scattering response for small aspect ratio nanorods, similar to the spherical nanoparticles but smaller in magnitude. However, the magnitude of this spectral shift decreases as the nanorod aspect ratio increases and the longitudinal plasmon resonance peaks at longer wavelengths. The transverse plasmon mode of the nanorods is either very weak or not observed at all by dark-field scattering spectroscopy. 
The measured nanorod absorption spectra are in very good agreement with the corresponding FDTD simulations shown in Fig. 4d-f, validating the procedure developed here. For the simulations, we neglect the substrate and instead employ an effective refractive index of $n_{\text {glycerol- }}$ glass $=1.495$. In order to perform simulations for the exact nanorod sizes, we correlate the optical measurements with scanning electron microscopy (SEM, see insets to Fig. 4a-c). The FDTD simulations are performed using linearly polarized light and exciting the nanorod along the long and short axis of the nanorod. We then again average the simulated spectra as in the case of the experiments. The simulations reproduce the spectral shapes and resonance maxima of both absorption and scattering well. In particular, the measured trend of decreasing spectral shift with larger aspect ratio is captured by the simulations. The shift between the absorption and scattering maxima is due to different frequency-dependent resonance conditions of the scattered and absorbed powers by the nanoparticles, as can be explained with a damped harmonic oscillator model proposed by Zuloaga et al. ${ }^{50}$

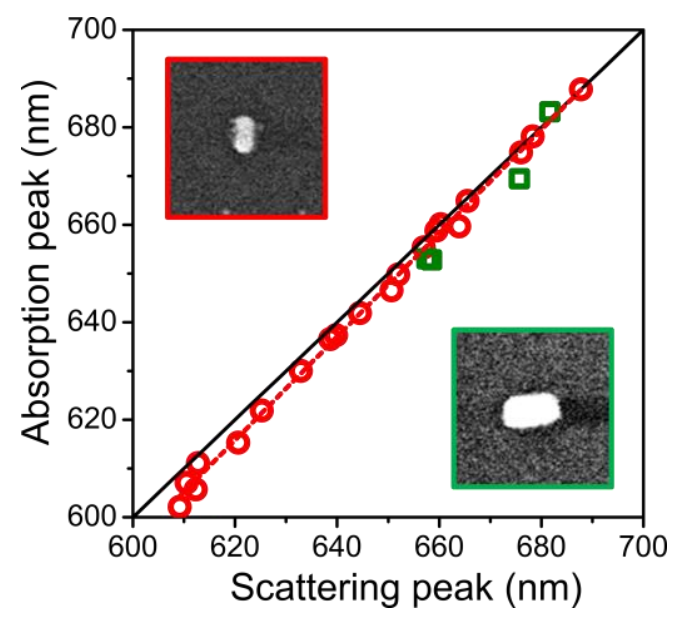

Figure 5. Correlation of longitudinal plasmon resonance absorption and scattering maxima for 20 small ( 0 ) and 4 large ( $\mathbf{a}$ ) volume nanorods. Example SEM images of a small and large 
volume nanorod are given as insets with red and green boxes, respectively. The size of each SEM image shown is $250 \mathrm{~nm}$. The solid black line (-) is a guide to the eye corresponding to no shift between absorption and scattering maxima. The dashed red line (---) is the linear fit to small volume nanorod data. A decreasing peak shift between absorption and scattering maxima of the longitudinal plasmon resonance is observed for larger aspect ratio nanorods.

To further quantify the shift between absorption and scattering maxima for the longitudinal plasmon resonance as a function of nanorod aspect ratio, we investigate a total of 24 single nanorods. Peak positions in the scattering and absorption spectra are determined by fitting Lorentzian curves. The scattering spectra are well fit with a Lorentzian line shape, signature of a single-particle measurement. For the absorption spectra, we only consider the longitudinal plasmon mode. We estimate an overall error for our measurements of less than $1 \mathrm{~nm}$. Fig. 5 plots the absorption maximum of each nanorod as a function of its corresponding scattering maximum. The magnitude of the spectral shift between longitudinal mode absorption and scattering decreases in a linear dependence with increasing nanorod aspect ratio (dashed line). The solid line indicates the case where absorption and scattering maxima are the same. The data in Fig. 5 includes measurements on two different nanorod samples, which differed in overall volume. Red points represent measurements on nanorods with an average width and length of $22 \pm 3 \mathrm{~nm}$, 49 \pm 6 nm (Fig. S15), while green points correspond to nanorods with an average width and length of $41 \pm 4 \mathrm{~nm}, 60 \pm 6 \mathrm{~nm}$ (Fig. S16). Color-coded, representative SEM images are given as in insets in Fig. 5. Despite a limited data set, the large volume nanorods appear to behave similar to the small volume nanorods. 
In summary, we have described a conceptually simple, efficient method to record singleparticle absorption spectra free from scattering by incorporating a broadband laser with photothermal microscopy. Implementation of an innovative calibration method to account for chromatic aberrations and varying excitation powers is necessary to obtain absorption spectra of anisotropic nanostructures with multiple plasmon modes. In particular, we have measured for the

first time the spectral shift between the absorption and scattering spectra of single gold nanoparticles as a function of size and shape. These results open the possibility of investigating pure absorption spectra of many other single nanostructures. We expect to be able to record absorption spectra of even smaller nanoparticles, and potentially single molecules, given the demonstrated sensitivity of photothermal imaging.

\section{ASSOCIATED CONTENT}

\section{Supporting Information}

Single-particle absorption spectroscopy; sample preparation; size distribution of nanoparticles with nominal diameters of $20 \mathrm{~nm}$ and $50 \mathrm{~nm}$; photothermal time trace to record the absorption spectrum; FDTD calculations; gold film thickness measured by AFM; pointing stability; material independence of calibration procedure; measured film spectrum at different spatial positions; corrected particle spectrum at different excitation angles; power dependence of nanoparticle absorption spectrum; power dependence of photothermal signal at different wavelengths; hyperspectral scattering spectroscopy and spatial correlation between absorption and scattering spectroscopy; microscope stability; reproducibility of the measured spectrum; size distribution of nanorods. This material is available free of charge via the Internet at http://pubs.acs.org 


\section{AUTHOR INFORMATION}

\section{Corresponding Author}

*Stephan Link, e-mail: slink@rice.edu

\section{Author Contributions}

The manuscript was written through contributions of all authors. All authors have given approval to the final version of the manuscript. $\$$ These authors contributed equally.

\section{Notes}

The authors declare no competing financial interest.

\section{ACKNOWLEDGMENT}

We acknowledge financial support from the Robert A. Welch Foundation (C-1664), the Office of Naval Research (N00014-10-1-0989), the Army Research Office (MURI W911NF-12-1-0407), the National Science Foundation (CHE-0955286), and a DURIP equipment grant (N00014-121.0727). M. Y. acknowledges financial support from the Richard E. Smalley Institute at Rice University through a Carl\&Lillian Illig Postdoctoral Fellowship. A. H. acknowledges support from the National Science Foundation through a Graduate Research Fellowship (0940902).

\section{ABBREVIATIONS}


AOTF, Acousto Optical Tunable Filter; PD, Photodiode; FDTD, Finite Difference Time

Domain; cw, continuous wave; SNR, signal-to-noise ratio; AFM, atomic force microscopy; SEM, scanning electron microscope. 


\section{REFERENCES}

(1) Orrit, M.; Bernard, J. Phys. Rev. Lett. 1990, 65, (21), 2716-2719.

(2) Moerner, W. E.; Orrit, M. Science 1999, 283, (5408), 1670-1676.

(3) Yildiz, A.; Forkey, J. N.; McKinney, S. A.; Ha, T.; Goldman, Y. E.; Selvin, P. R. Science 2003, 300, (5628), 2061-2065.

(4) Shields, A. J. Nat. Photon. 2007, 1, (4), 215-223.

(5) Haase, M.; Hubner, C. G.; Nolde, F.; Mullen, K.; Basche, T. Phys. Chem. Chem. Phys. 2011, 13, (5), 1776-1785.

(6) Zijlstra, P.; Orrit, M. Rep. Prog. Phys. 2011, 74, (10), 106401.

(7) Crut, A.; Maioli, P.; Del Fatti, N.; Vallee, F. Chem. Soc. Rev. 2014, 43, (11), 3921-3956.

(8) Olson, J.; Dominguez-Medina, S.; Hoggard, A.; Wang, L.-Y.; Chang, W.-S.; Link, S. Chem. Soc. Rev. 2015, 44, (1), 40-57.

(9) Lin, H. Z.; Tian, Y. X.; Zapadka, K.; Persson, G.; Thomsson, D.; Mirzov, O.; Larsson, P. O.; Widengren, J.; Scheblykin, I. G. Nano Lett. 2009, 9, (12), 4456-4461.

(10) Vogelsang, J.; Adachi, T.; Brazard, J.; Bout, D. A. V.; Barbara, P. F. Nat. Mater. 2011, 10, (12), 942-946.

(11) Gaiduk, A.; Yorulmaz, M.; Ruijgrok, P. V.; Orrit, M. Science 2010, 330, (6002), 353356.

(12) Celebrano, M.; Kukura, P.; Renn, A.; Sandoghdar, V. Nat. Photon. 2011, 5, (2), 95-98. 
(13) Chang, W. S.; Link, S. J. Phys. Chem. Lett. 2012, 3, (10), 1393-1399.

(14) Berciaud, S.; Cognet, L.; Tamarat, P.; Lounis, B. Nano Lett. 2005, 5, (3), 515-518.

(15) Chong, S. S.; Min, W.; Xie, X. S. J. Phys. Chem. Lett. 2010, 1, (23), 3316-3322.

(16) Selmke, M.; Braun, M.; Cichos, F. ACS Nano 2012, 6, (3), 2741-2749.

(17) Nedosekin, D. A.; Galanzha, E. I.; Dervishi, E.; Biris, A. S.; Zharov, V. P. Small 2014, $10,(1), 135-142$.

(18) Heylman, K. D.; Knapper, K. A.; Goldsmith, R. H. J. Phys. Chem. Lett. 2014, 5, (11), 1917-1923.

(19) Benkovic, S. J.; Hammes-Schiffer, S. Science 2003, 301, (5637), 1196-1202.

(20) Kukura, P.; Celebrano, M.; Renn, A.; Sandoghdar, V. J. Phys. Chem. Lett. 2010, 1, (23), $3323-3327$.

(21) Moerner, W. E.; Kador, L. Phys. Rev. Lett. 1989, 62, (21), 2535-2538.

(22) Carey, C. R.; Lebel, T.; Crisostomo, D.; Giblin, J.; Kuno, M.; Hartland, G. V. J. Phys. Chem. C 2010, 114, (38), 16029-16036.

(23) Billaud, P.; Marhaba, S.; Grillet, N.; Cottancin, E.; Bonnet, C.; Lerme, J.; Vialle, J. L.; Broyer, M.; Pellarin, M. Rev. Sci. Instrum. 2010, 81, (4), 043101.

(24) Havard, N.; Li, Z. M.; Murthy, V.; Lo, S. S.; Hartland, G. V. J. Chem. Phys. 2014, 140, (7), 074203. 
(25) McDonald, M. P.; Vietmeyer, F.; Aleksiuk, D.; Kuno, M. Rev. Sci. Instrum. 2013, 84, (11), 113104.

(26) Min, W.; Lu, S. J.; Chong, S. S.; Roy, R.; Holtom, G. R.; Xie, X. S. Nature 2009, 461, (7267), 1105-1109.

(27) Boyer, D.; Tamarat, P.; Maali, A.; Lounis, B.; Orrit, M. Science 2002, 297, (5584), 11601163.

(28) Berciaud, S.; Lasne, D.; Blab, G. A.; Cognet, L.; Lounis, B. Phys. Rev. B 2006, 73, (4), 045424.

(29) Gaiduk, A.; Ruijgrok, P. V.; Yorulmaz, M.; Orrit, M. Chem. Sci. 2010, 1, (3), 343-350.

(30) Selmke, M.; Heber, A.; Braun, M.; Cichos, F. Appl. Phys. Lett. 2014, 105, (1), 013511.

(31) Berciaud, S.; Cognet, L.; Poulin, P.; Weisman, R. B.; Lounis, B. Nano Lett. 2007, 7, (5), 1203-1207.

(32) Berciaud, S.; Cognet, L.; Lounis, B. Nano Lett. 2005, 5, (11), 2160-2163.

(33) Blancon, J. C.; Paillet, M.; Tran, H. N.; Than, X. T.; Guebrou, S. A.; Ayari, A.; San Miguel, A.; Phan, N. M.; Zahab, A. A.; Sauvajol, J. L.; Del Fatti, N.; Vallee, F. Nat. Commun. 2013, 4, 2542.

(34) Husnik, M.; Klein, M. W.; Feth, N.; Konig, M.; Niegemann, J.; Busch, K.; Linden, S.; Wegener, M. Nat. Photon. 2008, 2, (10), 614-617.

(35) Lindfors, K.; Kalkbrenner, T.; Stoller, P.; Sandoghdar, V. Phys. Rev. Lett. 2004, 93, (3), 037401 
(36) Muskens, O. L.; Del Fatti, N.; Vallee, F.; Huntzinger, J. R.; Billaud, P.; Broyer, M. Appl. Phys. Lett. 2006, 88, (6), 063109.

(37) Le Ru, E. C.; Etchegoin, P. G., In principles of surface enhanced Raman spectroscopy and related plasmonic effects. Elsevier: Amsterdam, 2009.

(38) Loo, C.; Lin, A.; Hirsch, L.; Lee, M. H.; Barton, J.; Halas, N. J.; West, J.; Drezek, R. Technol. Cancer. Res. T 2004, 3, (1), 33-40.

(39) Manjavacas, A.; Liu, J. G.; Kulkarni, V.; Nordlander, P. ACS Nano 2014, 8, (8), 76307638.

(40) Atwater, H. A.; Polman, A. Nat. Mater. 2010, 9, (3), 205-213.

(41) Mirin, N. A.; Bao, K.; Nordlander, P. J Phys Chem A 2009, 113, (16), 4028-4034.

(42) Sönnichsen, C.; Franzl, T.; Wilk, T.; von Plessen, G.; Feldmann, J.; Wilson, O.; Mulvaney, P. Phys. Rev. Lett. 2002, 88, (7), 077402.

(43) Dada, O. O.; Feist, P. E.; Dovichi, N. J. Appl. Optics 2011, 50, (34), 6336-6342.

(44) Franko, M.; Tran, C. D. Rev. Sci. Instrum. 1996, 67, (1), 1-18.

(45) Baffou, G.; Rigneault, H. Phys. Rev. B 2011, 84, (3), 035415.

(46) Bohren, C. F.; Huffman, D. R., Absorption and scattering of light by small particles. Wiley: New York, 1998.

(47) Hartland, G. V. Chem. Rev. 2011, 111, (6), 3858-3887.

(48) Wang, L.; Takeda, S.; Liu, C. C.; Tamai, N. J. Phys. Chem. C 2014, 118, (3), 1674-1681. 
(49) Tcherniak, A.; Ha, J. W.; Dominguez-Medina, S.; Slaughter, L. S.; Link, S. Nano Lett. 2010, 10, (4), 1398-1404.

(50) Zuloaga, J.; Nordlander, P. Nano Lett. 2011, 11, (3), 1280-1283. 
for TOC only

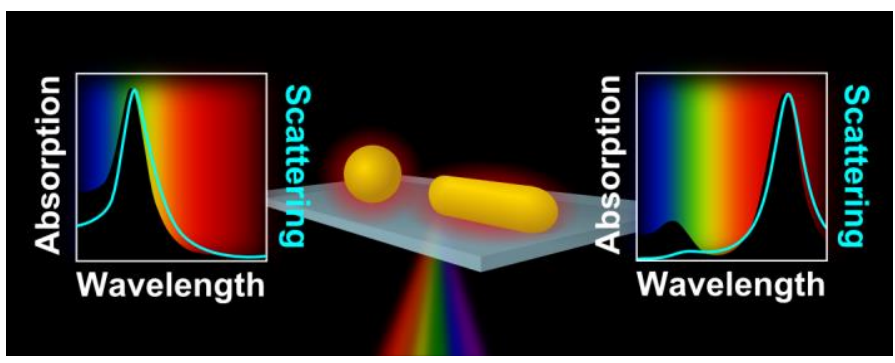

